\title{
ОБ ОДНОМ АЛГОРИТМЕ ПРИБЛИЖЕННОГО ВОССТАНОВЛЕНИЯ СИГНАЛОВ, ИСКАЖЕННЫХ ЛИНЕИНЫМ СГЛАЖИВАЮЩИМ ФИЛЬТРОМ
}

\author{
(Представил Я. Эйнасто)
}

\section{1. Постановка задачи}

Рассмотрим интегральное уравнение Фредгольма I рода с разностным ядром

$$
\int_{-\infty}^{\infty} k(x-y) u(y) \mathrm{d} y=v(x), \quad x \in(-\infty, \infty)
$$

или более кратко

$$
K u=v .
$$

Функция $v$ известна, требуется определить $u$. Ядро $k(x)$ предполагается неотрицательным, симметричным, двукратно дифференцируемым и нормированным к единице

$$
k(x) \geqq 0, \quad k(-x)=k(x), \quad k(x) \in C_{2}(-\infty, \infty), \quad \int_{-\infty}^{\infty} k(x) \mathrm{d} x=1 .
$$

Уравнение (1) допускает следующую интерпретацию: через сглаживающий фильтр $K$ пропускается сигнал $u$, на выходе регистрируется сглаженный сигнал v. По данным измерения сглаженного сигнала требуется определить истинный сигнал.

Уравнение (1) является некорректным по Адамару. Для его решения необходимо применить регуляризацию. Из существующих методов регуляризации наиболее известными являются метод оптимальной линейной фильтрации Винера $\left[{ }^{1}\right]$, метод Тихонова $\left[{ }^{2,3}\right]$, метод наиболее гладкого допустимого ансамбля Турчина $\left[{ }^{4}\right]$. Подробный обзор по методам решения уравнения (1) можно найти в [5].

В настоящей работе мы покажем, что

$$
u_{\tau}=v-\tau \frac{\mathrm{d}^{2}}{\mathrm{~d} x^{2}} K v
$$

является при оптимальном выборе параметра $\tau$ приближенным решением уравнения (1) в смысле

$$
E\left[\left\|u_{\tau}-u\right\|^{2}\right]<E\left[\|v-u\|^{2}\right] .
$$

Здесь $E[\cdot]$ - оператор математического ожидания, $\|\cdot\|$ - норма в метрике $L_{2}(-\infty, \infty)$, а $u$ представляет исходный (неизвестный, истинный) сигнал на входе фильтра. Параметр $\tau$ выражается через статистические моменты измеренного сигнала и погрешности измерения. В ряде случаев $\tau$ можно оценить с хорошей точностью через индивидуальную реализацию $v$. 
Формула (3) хорошо приближает истинный профиль в метрике $L_{2}$. Однако часто необходимо, чтобы восстановление имело одинаковую относительную точность во всем динамическом диапазоне изменения сигнала $u$.

Если $u$ и $v$ - положительные функции, то равномерной относительной точности восстановления можно добиться во многих случаях, используя следующую аппроксимирующую формулу

$$
u_{R}=v \exp \left[\left(u_{\tau}-u\right) / v\right] \text {, }
$$

где $u_{\tau}$ определяется формулой (3). Это решение практически совпадает с (3), если

$$
\left|u_{\tau}-v\right| \ll v,
$$

и вместе с тем может существенно улучшить относительную точность восстановления вблизи минимумов измеренного сигнала. Еще раз подчеркнем, что аппроксимация (5) применима только в случае положительных функций.

Основное внимание в настоящей работе уделяется обоснованию рещения (3) и, в частности, вопросу оптимального выбора параметра восстановления $\tau$. По своей направленности решение (3) относится к методам восстановления, при которых регуляризованный обратный оператор уравнения (1) $K_{R}^{-1}$ раскладывается в ряд по степеням дифференциального оператора $\mathrm{d}^{2} / \mathrm{d} x^{2}$ (по поводу представления $K_{R}^{-1}$ в виде ряда по дифференциальным операторам см. $\left.\left[{ }^{6,7}\right]\right)$.

\section{2. Оптимальный выбор параметра восстановления}

Определим $\tau$ из условия минимальности функционала

$$
Q(\tau)=2 \pi E\left[\left\|u_{\tau}-u\right\|^{2}\right]=2 \pi E\left[\int\left(u_{\tau}-u\right)^{2} \mathrm{~d} x\right] .
$$

Предполагается, что исходные профили $u$, а также ошибки измерения образуют статистический ансамбль. Минимизация $Q$ по $\tau$ гарантирует близость (наилучшее приближение в метрике $L_{2}$ ) восстановленного решения $u_{\tau}(3)$ к истинному сигналу по крайней мере в среднеквадратичном смысле. Предлагаемый способ оптимизации близок к методу оптимальной фильтрации Винера $\left[{ }^{1}\right]$. Обозначим

Для решения задачи удобно использовать преобразование Фурье.

$$
\begin{gathered}
\tilde{k}(\omega)=F k(x) \equiv \int e^{i \omega x} k(x) \mathrm{d} x, \\
\tilde{u}(\omega)=F u(x), \quad \tilde{v}(\omega)=F v(x), \quad \tilde{u}_{\tau}(\omega)=F u_{\tau}(x) .
\end{gathered}
$$

Для дальнейшего изложения необходимо в уравнении (1) явно выделить погрешность измерения в измеренном сигнале, т. е.

$$
v=K u+\delta \text {. }
$$

Преобразование Фурье для этого уравнения будет

$$
\tilde{v}(\omega)=\tilde{v}_{0}(\omega)+\tilde{\delta}(\omega),
$$

где

$$
\widetilde{v}_{0}(\omega)=\widetilde{k}(\omega) \tilde{u}(\omega),
$$

а $\tilde{\delta}(\omega)=F \delta(x)$ - преобразование Фурье для погрешности. Формула (3) приводится к виду

$$
\tilde{u}_{\tau}(\omega)=\left[1+\tau \omega^{2} \tilde{k}(\omega)\right] \tilde{v}(\omega),
$$

а функционал $Q(\tau)-$ к виду

$$
Q(\tau)=E\left[\int\left|\tilde{u}_{\tau}(\omega)-\tilde{u}(\omega)\right|^{2} \mathrm{~d} \omega\right] .
$$




$$
E\left[v_{0}(x) \delta\left(x_{1}\right)\right]=0
$$

(это условие выполняется, например, если $u$ и $v$ являются статистически независимыми и $E[\delta(x)]=0)$. Тогда для $Q(\tau)$ получим представление

где

$$
Q(\tau)=Q_{0}-2 Q_{1} \tau+Q_{2} \tau^{2},
$$

$$
\begin{gathered}
Q_{0}=\int E\left[|\tilde{v}(\omega)-\tilde{u}(\omega)|^{2}\right] \mathrm{d} \omega, \\
Q_{1}=\int \omega^{2}\left\{E\left[\left|\tilde{v}_{0}\right|^{2}\right]-\tilde{k}(\omega) E\left[\left|\tilde{v}_{0}(\omega)\right|^{2}+|\tilde{\delta}(\omega)|^{2}\right]\right\} \mathrm{d} \omega, \\
Q_{2}=\int \omega^{4} \tilde{K}^{2}(\omega) E\left[\left|\tilde{v}_{0}(\omega)\right|^{2}+|\tilde{\delta}(\omega)|^{2}\right] \mathrm{d} \omega .
\end{gathered}
$$

При выводе этих формул использованы соотношения

$$
\bar{k}(\omega)=\widetilde{k}(-\omega)=\widetilde{k}(\omega),
$$

вытекающие из вещественности и симметричности ядра $k(x)$.

Минимум $Q(\tau)$ находится в точке

$$
\tau=\tau_{e}=Q_{1} / Q_{2} .
$$

Полученное значение параметра $\tau$ не только минимизирует $Q(\tau)$, но и дает улучшение восстановленного профиля (3) по сравнению с измеренным сигналом $v$, если только $\tau_{e} \neq 0$. Истинность этого утверждения следует из неравенства

$$
E\left[\left\|u_{\tau_{e}}-u\right\|^{2}\right]=\frac{1}{2 \pi} Q\left(\tau_{e}\right)<\frac{1}{2 \pi} Q(0)=\frac{1}{2 \pi} Q_{0}=E\left[\|v-u\|^{2}\right] .
$$

Таким образом, формула (3) при $\tau=\tau_{e}$ представляет собой регуляризованное решение уравнения (1).

\section{3. Оценка параметра восстановления по результатам единичного измерения}

Для использования формул (14)-(16) нужно знать вторые одноточечные моменты $E\left[\left|\tilde{v}_{0}\right|^{2}\right]$ и $E\left[|\tilde{\delta}|^{2}\right]$. Эти моменты значительно варьируют от задачи к задаче, поэтому здесь могут возникать самые разнообразные ситуации. Один из наиболее часто встречаемых случаев будет разобран ниже.

Предположим, что

1. Случайный ансамбль $\left\{v_{0}\right\}$ состоит из одной функции

$$
E\left[\left|\widetilde{v}_{0}(\omega)\right|^{2}\right]=\left|\tilde{v}_{0}(\omega)\right|^{2},
$$

так что статистические свойства измеряемой величины $v=v_{0}+\delta$ полностью определяются свойствами погрешности $\delta$.

2. Основной вклад в интегралы (14), (15) дает область $\omega \in[-\Omega, \Omega]$. Таким образом, интегрирование в бесконечных пределах можно заменить интегрированием в конечной области $[-\Omega, \Omega]$.

3. В области $[-\Omega, \Omega]$ выполняется неравенство

$$
\left|\tilde{v}_{0}(\omega)\right|^{2} \gg E\left[|\tilde{\delta}(\omega)|^{2}\right], \quad|\omega|<\Omega,
$$

что дает право воспользоваться приближениями

$$
\left|\tilde{v}_{0}(\omega)\right|^{2} \cong|\tilde{v}(\omega)|^{2}, \quad\left|\widetilde{v}_{0}(\omega)\right|^{2}+E\left[|\tilde{\delta}(\omega)|^{2}\right] \cong|\tilde{v}(\omega)|^{2}, \quad|\omega|<\Omega .
$$

При сделанных предположениях из формул (14) и (15) получим следующие оценочные выражения для $Q_{1}$ и $Q_{2}$ 


$$
Q_{1}=2 \int_{0}^{\Omega} \omega^{2}[1-\widetilde{k}(\omega)]|\tilde{v}(\omega)|^{2} \mathrm{~d} \omega, \quad Q_{2}=\left.2 \int_{0}^{\Omega}\left|\omega^{4} \tilde{k}^{2}(\omega)\right| \tilde{v}(\omega)\right|^{2} \mathrm{~d} \omega .
$$

Преимущество этих формул в том, что входящие сюда функция $\tilde{v}(\omega)$ и параметр $\Omega$ определяются по данным единичного эксперимента. Таким образом, формулы (20) позволяют оценить параметры $Q_{1}$ и $Q_{2}$ для каждого конкретного профиля $v(x)$, или другими словами: каждой функции $v(x)$ формулами (16) и (20) ставится в соответствие свой параметр регуляризации $\tau$.

Наиболее ответственный момент в описанном приближенном алгоритме - проверка условия (18). Это условие обычно хорошо выполняется, если преобразование Фурье погрешности $\tilde{\delta}(\omega)$ является стационарным случайным процессом

$$
E\left[(\dot{\delta}(\omega))^{2}\right]=\varepsilon^{2}=\text { const. }
$$

и имеет место неравенство

$$
|\tilde{v}(0)|^{2} \gg \varepsilon^{2}
$$

\section{4. Асимптотика параметра восстановления для гладких функций}

Предположим, что в интегралах (20) функция $|\widetilde{v}(\omega)|^{2}$ быстро стремится к нулю при $|\omega| \rightarrow \infty$ по сравнению с функцией $\widetilde{k}(\omega)$. Это означает, что спектр измеренного сигнала значительно уже спектральной ширины фильтра и $v(x)$ - гладкая, медленноменяющаяся по сравнению с $k(x)$, функция. В таком случае в формулах (20) можно приближенно полагать

$$
1-\widetilde{k}(\omega) \cong \sigma^{2} \omega^{2} / 2, \quad \widetilde{k}^{2}(\omega) \cong 1,
$$

где

$$
\sigma^{2}=\int_{-\infty}^{\infty} x^{2} k(x) \mathrm{d} x
$$

- дисперсия фильтра $K$.

Как следствие из (17), (21) получим

$$
\tau \cong \sigma^{2} / 2 \text {. }
$$

Это и есть асимптотика параметра восстановления в случае гладких функций $v$, или, что то же самое, - при стремлении разрешающей способности фильтра $K$ к бесконечности (при $\delta \rightarrow 0)$.

\section{5. Численные примеры}

Мы показали, что приближенное регуляризованное решение (3), (14)-(16) представляет собой частично восстановленный профиль, т. е. что имеет место неравенство (4). Однако по этому неравенству нельзя судить, насколько существен эффект восстановления и не возникают ли при этом такие нежелательные побочные эффекты, как осцилляция, острые всплески в восстановленном профиле и т. п. На эти вопросы весьма затруднительно дать однозначный ответ, так как вид сглаживающего оператора и характер входного сигнала $u$ могут значительно варьировать от задачи к задаче. По этой причине необходима проверка алгоритма в каждом конкретном случае.

Ниже приводятся результаты численного моделирования восстановления яркости горизонта Земли $u(x)$ по сглаженным данным $v(x)$, полученным при измерениях из космоса.

Аргумент $x$ представляет собой в нашем примере перигейную высоту оптической оси приемника излучения над геометрическим гори- 
зонтом. Сглаживающее воздействие приемника моделирует гауссовый фильтр:

$$
k(x)=\frac{1}{\sqrt{2 \pi} \sigma} \exp \left[-x^{2} / 2 \sigma^{2}\right]
$$

где $\sigma-$ параметр, определяющий величину поля зрения прибора.

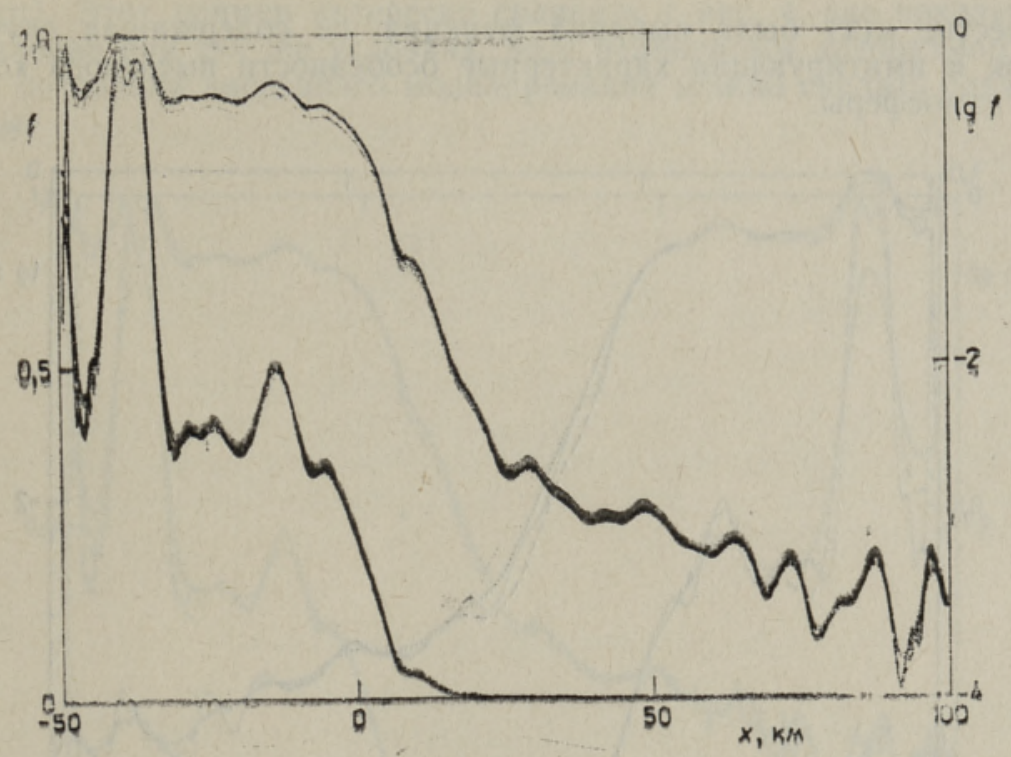

Рис. 1. Моделирование восстановления по формуле (3); $\sigma=1$ км, $\varepsilon_{0}=0$; сплошная кривая - исходный профиль, пунктир - сглаженный сигнал, штриховая линия восстановленный профиль. Верхняя группа кривых - логарифмический масштаб, нижняя группа кривых - линейный масштаб по оси $y$.

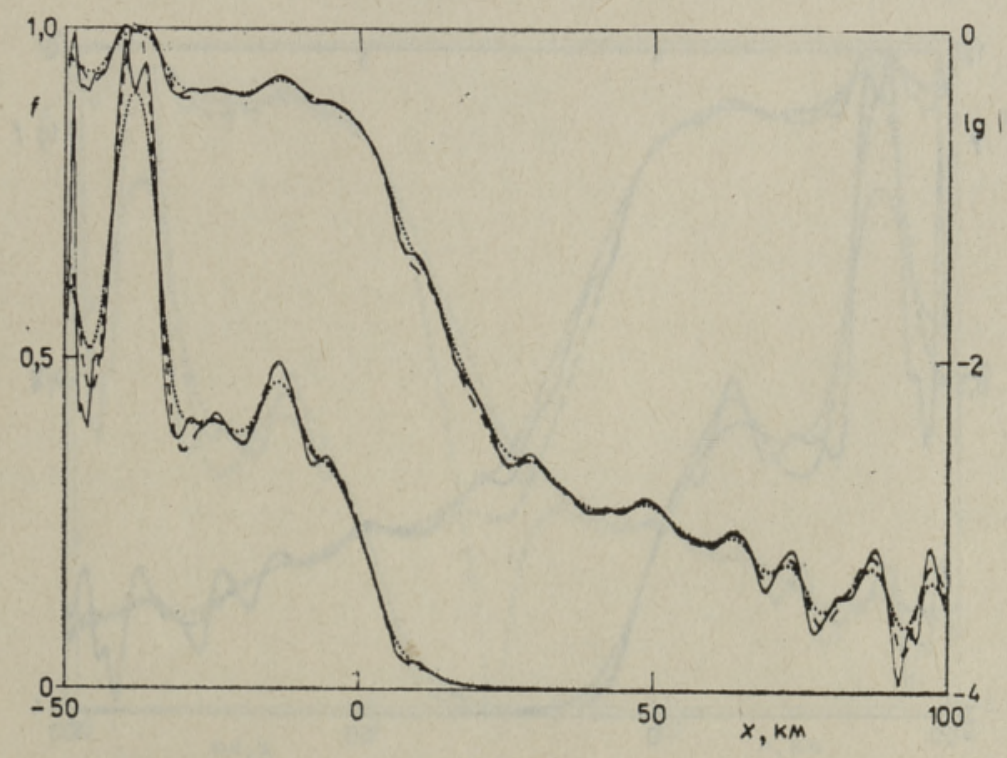

Рис. 2. Моделирование восстановления по формуле (3); $\sigma=2$ км, $\varepsilon_{0}=0$ (обозначеения см, на рис. 1). 
Моделирование происходило по схеме

$$
u\left(x_{i}\right) \rightarrow v\left(x_{i}\right)=K u\left(x_{i}\right)+\delta\left(x_{i}\right) \rightarrow u_{\tau}\left(x_{i}\right) \rightarrow u_{R}\left(x_{i}\right),
$$

где $x_{i}$ - равномерная сетка с шагом 0,5 км. Погрешность $\delta\left(x_{i}\right)$ моделировалась некоррелированным нормально-распределенным случайным вектором с параметрами

$$
E\left[\delta\left(x_{i}\right)\right]=0, \quad E\left[\delta\left(x_{i}\right) \delta\left(x_{j}\right)\right]=\varepsilon_{0}^{2} \delta_{i j} .
$$

В качестве $u(x)$ была выбрана функция, не содержащая случайных ошибок и имитирующая характерные особенности высотного хода яркости атмосферы.

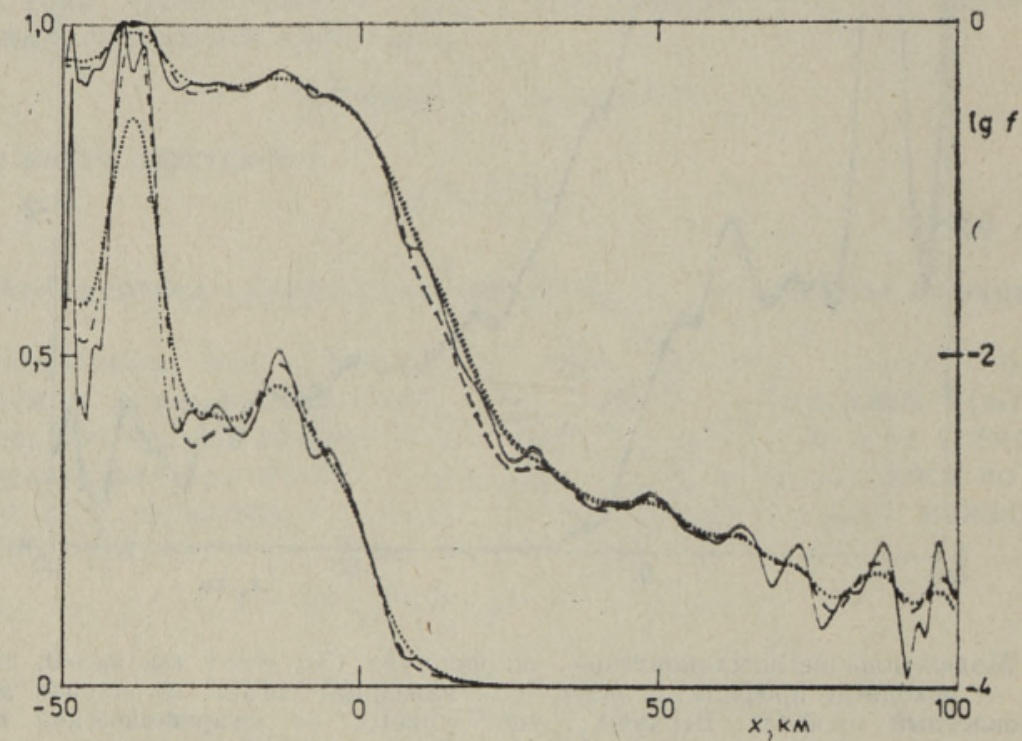

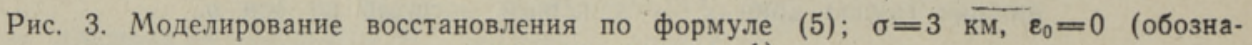
чения см. на рис. 1).

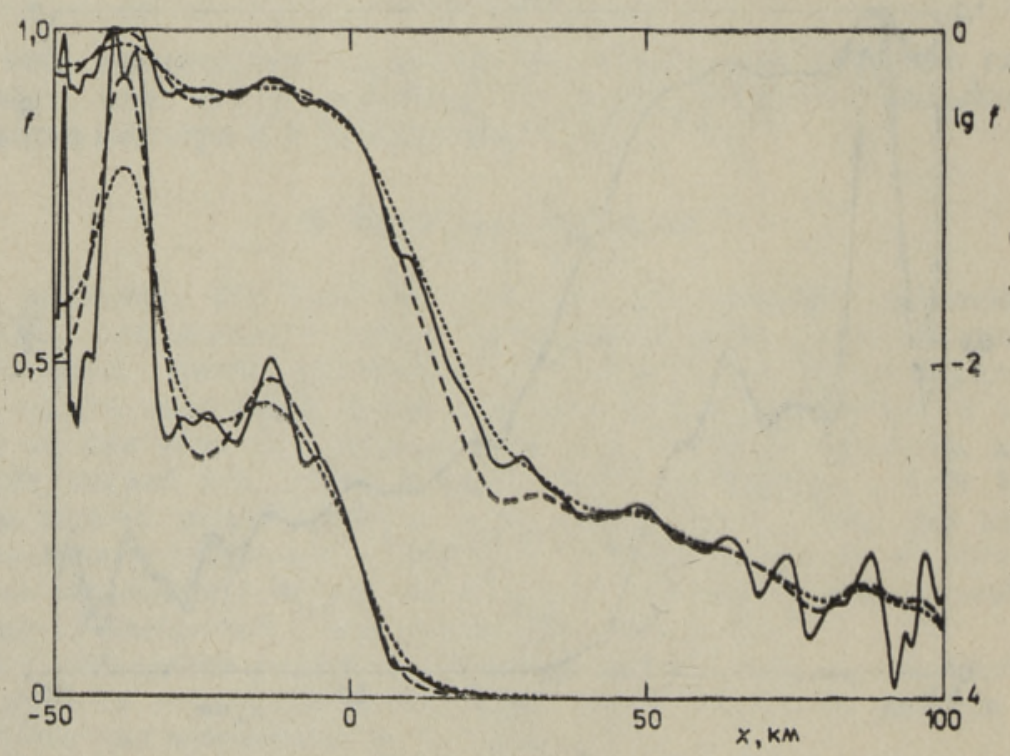

Рис. 4. Моделирование восстановления по формуле (5); $\sigma=4$ км, $\varepsilon_{0}=0$ (обозначения см. на рис. 1). 
Все встречающиеся в алгоритме производные и интегралы вычислялись с использованием дискретного преобразования Фурье.

На рис. $1-4$ показаны результаты моделирования для $\sigma=1,2,3$ и 4 км при $\delta(x)=0$. На рис. 1 и 2 восстановление произведено по формуле (3), на рис. 3 и $4-$ по формуле (5). На рис. 5 показано влияние высокочастнотного шума в измеренном сигнале $\left(\varepsilon_{0}=0,0005\right)$ на результаты восстановления при $\sigma=2$ км. Наконец, рис. 6 иллюстрирует действие линейного алгоритма (3) при сильном сглаживании ( $\sigma=$ $=4$ км). Этот пример интересно сравнить с рис. 4 , где показано восстановление при тех же условиях, но с нелинейным алгоритмом (5).

На основании численного моделирования можно сделать следующие выводы:

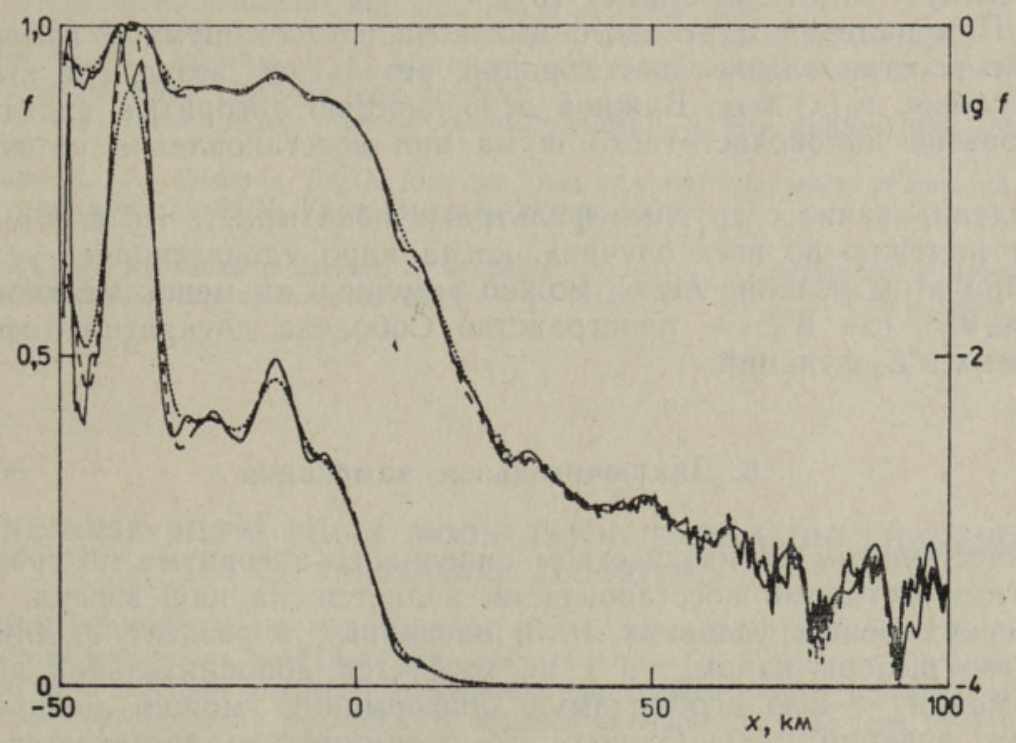

Рис. 5. Моделирование восстановления по формуле (3); $\sigma=2$ км, $\varepsilon_{0}=0,0005$ (обозначения см. на рис. 1).

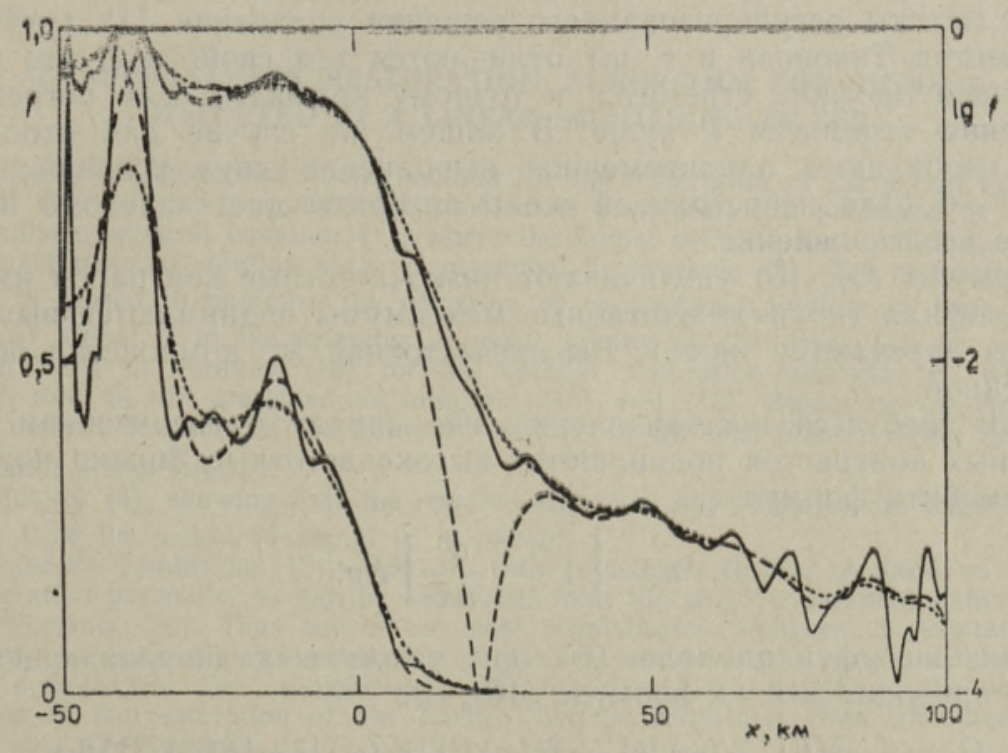

Рис. 6. Моделирование восстановления по формуле (3); $\sigma=4$ км, $\varepsilon_{0}=0$ (обозначения см. на рис. 1). 
1. С возрастанием сглаживающего воздействия прибора, т. е. при увеличении $\sigma$, уровень восстановления понижается. Однако в любом случае восстановленный профиль $\left(u_{\tau}\right.$ или $\left.u_{R}\right)$ лучше приближает в метрике $L_{2}$ истинный профиль, чем измеренная функция $v(x)$.

2. С увеличением $\sigma$ в восстановленном профиле пропадают мелкие детали, крупномасштабные же особенности исходного профиля восстанавливаются относительно хорошо.

3. При малых б восстановление по формулам (3) и (5) приводит к близким результатам. При больших $\sigma$ более качественное восстановление дает формула (5). Различие может стать заметным вблизи острых минимумов функции $v$. Поэтому в тех случаях, когда нужно добиться равномерной относительной точности восстановления, предпочтение следует отдать алгоритму (5).

4. При наличии адитивного высокочастотного шума в измеренном сигнале восстановление дает хорошие результаты везде, где выполняется условие $v_{0}(x) \gg \varepsilon_{0}$. Важной особенностью алгоритма является то, что уровень высокочастотного шума при восстановлении не усиливается.

Моделирование с другими фильтрами показывает, что алгоритм работает надежно во всех случаях, когда ядро удовлетворяет условиям (2). При этом условие $k \in C_{2}$ можно заменить на менее жесткое условие $k \in W_{2}^{2}$, где $W_{3}{ }^{2}$ - пространство Соболева двукратно дифференцируемых в $L_{2}$ функций.

\section{6. Заключительные замечания}

Существенным преимуществом описанного алгоритма по сравнению с другими методами восстановления является, на наш взгляд, то, что при весьма общих условиях $1-3$, описанных в разделе 3 , для оптимального выбора параметра $\tau$ не требуется дополнительной внешней информации - всю необходимую информацию можно получить из спектра функции $v(x)$. Однако это преимущество достигается ценой приближенного характера самого алгоритма.

Приближенность решения (3) заключается в следующем. Классические методы регуляризованного решения уравнения (1) (метод Винера, метод Тихонова и т. п.) отличаются тем свойством, что восстановленный профиль стремится к точному профилю, если погрешности измерения стремятся к нулю. В нашем же случае для сходимости $u_{\tau} \rightarrow u$ необходимо одновременное выполнение двух условий $\sigma \rightarrow 0$ и $d^{2} v / d x^{2} \rightarrow 0$. Для непостоянной же $v$ алгоритм дает заведомо приближенное восстановление.

Формулы (3), (5) увеличивают низкочастотные контрасты измеренного профиля (крупномасштабные максимумы поднимаются выше, минимумы опускаются ниже). Высокочастотная же компонента остается неизменной.

Если требуется восстановление, где наряду с увеличением низкочастотных контрастов подавляются высокочастотные, можно попытаться применить формулу

$$
u_{\tau}=\left[1-\tau \frac{\mathrm{d}^{2}}{\mathrm{~d} x^{2}}\right] K v
$$

Минимизация функционала (6) при таком восстанавливающем операторе приводит для $\tau$ к формуле (16), где

$$
Q_{1}=\int \omega^{2}\left\{E\left[\left|\tilde{v}_{0}(\omega)\right|^{2}\right]-\tilde{k}^{2}(\omega) E\left[\left|\tilde{v}_{0}(\omega)\right|^{2}+|\tilde{\delta}(\omega)|^{2}\right]\right\} \mathrm{d} \omega,
$$

a $Q_{2}$ определяется соотношением (15). Как показывает численное мо- 
делирование, формула (23) приводит к не менее хорошим результа̀там, чем формула (3). Однако пока доказать, что решение (23) удовлетворяет критерию восстановления (4), не удалось.

\section{ЛИТЕРА Т У РА}

1. Wiener, $N$. Extrapolation, Interpolation and Smoothing of Stationary Time Series with Engineering Applications. New York, Wiley, 1949.

2. Тихонов А. Н., Арсенин В. Я. Методы решения некорректных задач. М., Наука, 1979.

3. Тихонов А. Н., Гончаровский А. В., Степанов В. В., Ягола А. Г. Регуляризующие алгоритмы и априорная информация. М., Наука, 1983.

4. Турчин В. Ф. // Ж. вычнсл. мат. и мат. физ., 1967, 7, № 6, 1270-1284.

5. Верлань А. Ф., Сизиков В. С. Интегральные уравнения: методы, алгоритмы программы. Киев, Наукова думка, 1986.

6. Латтес Р., Лионс Ж.-Л. Метод квазиобращения и его приложения. М., Мир, 1970.

7. Верлань А. Ф., Сизиков В. С. Методы решения интегральных уравнений с программами для ЭВМ. Киев, Наукова думка, 1978.

Ннститут астрофизики и физики атмосферы Академии наук Эстонской ССР
Поступила в редакцию 30/III 1988

\section{R. $R O O M$}

\section{LINEAARSE FILTRI POOLT MOONUTATUD SIGNAALIDE LIGIKAUDSE TAASTAMISE ALGORITM}

Artikkel käsitleb Gaussi tüüpi filtriga silutud signaalide taastamist. Selline ülesanne tekib sageli optikas, kosmilises kaugseires ja mujal. On kirjeldatud üht arvutuslikult lihtsat ja kiiret meetodit probleemi ligikaudseks lahendamiseks ning näidatud, et saadav lähendlahend aproksimeerib signaali filtri sisendis paremini kui seda teeb filtri väljundis registreeriv signaal. See tähendab, et algoritm on tõesti taastav.

\section{R. $R O O M$}

\section{AN APPROXIMATE RESTORATION ALGORITHM FOR SIGNALS DISTORTED BY A LINEAR SMOOTHING FILTER}

A simple and numerically quick method for the restoration of the signal $u(x)$ from smoothed data $v(x)$ is proposed. The problem is mathematically presented as the first kind Fredholm integral equation (1), where the kernel satisfies the conditions (2). The approximate restored solution $u_{\tau}(x)$ is presented by Formula $(3)$. The restoration parameter $\tau$ is chosen to minimize the deviation of the restored solution $u_{\tau}$ from the real (unknown) signal $u$ in the meaning of mean squares in the space $L_{2}(-\infty, \infty)$. This condition leads to Formula (16) for the optimal restoration-parameter $\tau_{e}$, where the terms $Q_{1}$ and $Q_{2}$ are presented as integrals (14) and (15), depending on the second order statistical momenta of the Fourier transform of the true filtered signal $\widetilde{v}_{0}(\omega)$ and experimental errors $\tilde{\delta}(\omega)$.

Inequality (4), showing that the restored signal $u_{\tau}$ approximates the original signal $u$ better than the measured signal $v$, is proved.

In case the conditions (17)-(19) are valid, the terms $Q_{1}$ and $Q_{2}$ (and, as a result, the restoration parameter $\tau$ ) can be calculated from the single-experiment data with the help of Formula (20). Thus one of the most sophisticated problems of restoration, i. e. how to choose optimally the restoration parameter, is solved in a simple manner.

To demonstrate the possibilities of the algorithm, the results of the numerical simulation of the restoration of the Earth's horizon brightness from smoothed remote sensing data are presented. 